\title{
Withdrawal of life support in babies: deceptive signals
}

\author{
M L Chiswick
}

Even an ardent 'pro-life' supporter might agree that when a baby has clearly entered the process of dying, and when continuation of ventilatory support is obviously pointless, then it is preferable to allow nature to take its course.

Others hold the view that 'quality of life' is also a consideration. Though the expression has many shades of meaning, those who favour withdrawing life support on the grounds of 'quality of life' agree that the decision must be jointly made with parents. It is the consultant, the doctor who is responsible for directing the care of the baby (and who therefore ultimately takes the responsibility for initiating life support), who must make this decision with the parents. The need to identify a senior person when making decisions is one reason why all babies in maternity units, whether or not admitted to a neonatal unit, should be under the care of a named consultant.

Though the ethics of withdrawing life support from chronically ill newborn babies have been discussed many times, little attention has been given to the circumstances of a baby's care that might prompt a consultant to consider that course of action. When confronted with sensitive issues, nurses and junior doctors often signal their feelings in subtle ways. Although consultants must be receptive to these messages-and indeed may have already begun to question privately the wisdom of continuing life support-early impressions are liable to be deceptive and charged with personal emotion rather than reflect objective and thoughtful appraisal. If we, with parents, are to act 'in the best interests' of the baby we must understand certain pitfalls before initiating discussion with parents.

The following points overlap to some extent, but they are discussed separately because each has rather different implications.

\section{Despair}

The frustration felt by staff during the prolonged care of a baby whose course is punctuated by repeated episodes of deterioration and improvement is well known. Despair is an inconsistent emotion. It varies from one member of staff to the next, and is powerfully influenced by the overall amount of stress in the unit. During prolonged periods of stress, perhaps generated by a large work load, despair among staff often becomes endemic and can lead to a consensus opinion that withdrawal of ventilatory support should be a consideration.

For example, a chronically sick baby might 'collapse' (deteriorate acutely) during mechanical ventilation-perhaps as a result of failure to recognise promptly a blocked or misplaced endotracheal tube. After resuscitation, and when the baby is settled once more on the ventilator, attention might be drawn for the first time to the question of withdrawing ventilatory support. The wisdom of resuscitating the baby is, in hindsight, questioned. It may well be that one or more prolonged periods of circulatory failure will indeed compromise long term outcome, but that is an issue which must be judged on its own merit and as scientifically as possible. What must be avoided is bias about the long term or short term prognosis being engendered by the collapse itself, which is a reversible occurrence. Neither staff despair nor parental despair are proper reasons for withdrawing life support.

\section{Appearance of the baby}

Sometimes the ravages of chronic neonatal morbidity are reflected in a baby's appearance. I refer here to features that command attention by virtue of their unpleasant and distasteful impact, which cannot be promptly alleviated, and which call to mind the notion of 'suffering'. For example, it may be impossible to meet the energy needs of certain chronically ill babies, and this leads to the appearance of malnutrition. Superimposed on this may be the green-yellow discolouration of the skin in chronic obstructive liver disease associated with parenteral nutrition, and the marks of trauma from surface electrodes and old intravenous infusion sites. However distasteful a baby's appearance may be to staff and parents alike we should strive to disassociate this from considerations of withdrawing life support.

\section{Parents who do not visit}

One stimulus for continuing the care of a chronically sick baby is the presence of parents beside the cot. With few exceptions, parents who are concerned about their chronically sick baby encourage the staff to carry on and this feeling of shared responsibility ('we are all in it together') is important. There may be understandable reasons why parents do not visit, but when the element of sharing is not there the incentive for continuing life support may be weakened earlier than it might otherwise be. Our requirement to act in the best interest of the baby applies whether or not his parents are seen to be concerned. 


\section{Biased impression of prognosis}

There are three main concerns when considering the withdrawal of ventilatory support: the extent to which the baby is perceived to be suffering; the baby's chances of survival; and the anticipated long term outcome should the baby survive. The notion of 'suffering', which is certainly open to biased impressions, is beyond the scope of this short paper.

Given a moral stance that permits withdrawal of life support, the main factor that prompts caution, in my experience, is uncertainty about the short term and long term outcomes. The perception of a baby's prognosis is open to bias in a number of ways. The features of chronic neonatal morbidity, particularly in preterm babies, reflect a stereotyped array of clinical problems. A recent experience of a baby's death, despite prolonged intensive care, may be erroneously used as a yardstick to judge another baby's chances of survival ('It's pointless, he's just the same as baby $\mathbf{X}$ and all our care made no difference'). It may be that a baby does indeed have a poor prognosis, but withdrawal of life support should not be based on comparisons with another baby who recently died.

Another source of bias is the interpretation of abnormalities that are seen on ultrasound brain scans. Imagine two babies, both aged 6 weeks, and both with moderate but non-progressive ventriculomegaly diagnosed by brain scan. Ventriculomegaly in the baby no longer requiring ventilatory support may be judged to carry a reasonably good prognosis. In contrast, the same degree of ventriculomegaly in a baby who is receiving assisted ventilation for chronic lung disease may be used to question the wisdom of continuing life support ('he's already got some cerebral atrophy'). Now it may well be that the baby who is more ill does have cerebral atrophy, which might be a real consideration. If so, this interpretation must be made thoughtfully with due regard to the accuracy of diagnosis and implications for prognosis based on scientific observations. We should avoid the double standard whereby the same structural abnormality is used as a pointer towards later severe morbidity in an ill baby but not in a well baby.

If, with parents, we are to act in the best interests' of the baby we must acknowledge that subjectivity is not always easy to avoid. Erecting procedural safeguards, such as hospital ethics committees, is not the answer. The solution, in so far as there is one at all, rests with circumspection, self analysis, and the recognition of deceptive signals before voicing an argument for the withdrawing of life support. From the outset we should ask ourselves:

- Am I being driven by despair?

- Am I being unduly influenced by the external appearance of the baby?

- Would I think differently if the parents had been beside the cot every day?

- Are my predictions about outcome based on current scientific knowledge? 